\title{
Malignant Triton Tumor in the Abdominal Wall: A Case Report
}

\author{
Yoshiyuki Kitamura ${ }^{1}$, Koichiro Abe ${ }^{1}$, Shingo Baba ${ }^{1}$, Takuro Isoda ${ }^{1}$, \\ Yasuhiro Maruoka ${ }^{1}$, Yoshio Matsuo ${ }^{1}$, Yuichiro Kubo ${ }^{2}$, Akio Sakamoto ${ }^{3}$, \\ Hiroshi Nishimura ${ }^{4}$, Masayuki Sasaki ${ }^{5}$, Hiroshi Honda ${ }^{1}$ \\ ${ }^{1}$ Department of Clinical Radiology, Kyushu University Hospital, Fukuoka, Japan \\ ${ }^{2}$ Department of Anatomic Pathology, Pathological Sciences, Graduate School of Medical Sciences, \\ Kyushu University, Fukuoka, Japan \\ ${ }^{3}$ Department of Orthopaedic Surgery, Kyushu University Hospital, Fukuoka, Japan \\ ${ }^{4}$ Department of Clinical Radiology, Saiseikai Futsukaichi Hospital, Fukuoka, Japan \\ ${ }^{5}$ Department of Health Sciences, Graduate School of Medical Sciences, Kyushu University, Fukuoka, Japan \\ Email: k-abe@radiol.med.kyushu-u.ac.jp
}

Received March 6, 2013; revised April 6, 2013; accepted April 15, 2013

Copyright (C) 2013 Yoshiyuki Kitamura et al. This is an open access article distributed under the Creative Commons Attribution License, which permits unrestricted use, distribution, and reproduction in any medium, provided the original work is properly cited.

\begin{abstract}
Malignant triton tumor (MTT) is a rare variant of malignant peripheral nerve sheath tumor (MPNST) with rhabdomyosarcomatous differentiation. We report the case of a 54-year-old male without a history of neurofibromatosis type 1 (NF1) who had a growing abdominal wall tumor diagnosed as MTT. Computed tomography (CT), magnetic resonance imaging (MRI) and 2-[F-18]-fluoro-2-deoxy-D-glucose positron emission tomography/CT (FDG-PET/CT) were performed. The MRI and FDG-PET/CT indicated that the lateral component of the tumor was composed of many proliferative cells, corresponding to the histopathological finding of a cellular proliferation of spindle-shaped cells. In light of this case and previous reports, it is apparent that FDG-PET/CT is a helpful tool for distinguishing MTT from benign peripheral nerve sheath tumor.
\end{abstract}

Keywords: Malignant Triton Tumor (MTT); Malignant Peripheral Nerve Sheath Tumor (MPNST); 2-[F-18]-Fluoro-2-Deoxy-D-Glucose Positron Emission Tomography/Computed Tomography (FDG-PET/CT)

\section{Introduction}

Malignant triton tumor (MTT) is a variant of malignant peripheral nerve sheath tumor (MPNST) with rhabdomyosarcomatous differentiation. It is a rare tumor constituting 5\% of all MPNSTs [1], and about two-thirds of MTT arise in patients with neurofibromatosis type 1 (NF1) [2].

Computed tomography (CT) and magnetic resonance imaging (MRI) are useful for detecting the localization and delineating the extent of MPNST including MTT, and 2-[F-18]-fluoro-2-deoxy-D-glucose (FDG) accumulation reflects their biological activities.

To date, FDG-positron emission tomography (FDGPET) findings of MTT have been demonstrated in only three reports [3-5], to the best of our knowledge. Although several studies reported that FDG-PET was a sensitive and specific tool for distinguishing MPNST from benign peripheral nerve sheath tumor [5-10], there has been very little discussion about the details of the radiological features of MTTs.

We present the case of a patient who was found to have an MTT arising in the abdominal wall, and we report the imaging findings obtained by $\mathrm{CT}$, MRI, and FDG-PET/CT.

\section{Case Report}

A 54-year-old male was admitted to our hospital after a 2-year history of an enlarging right upper abdominal wall tumor. He had no pain or other symptoms and no family history of NF1. A tumor about $10 \mathrm{~cm}$ in dia. was palpable on the right side of the lower chest to the upper abdomen. There was no skin reddening, skin ulcer, bleeding or tenderness. The blood examination results included a white blood cell count of $8720 / \mu 1$ and C-relative protein level of $1.27 \mathrm{mg} / \mathrm{dl}$, indicating the existence of slight inflammation. Total cholesterol $(284 \mathrm{mg} / \mathrm{dl})$ was slightly elevated but all other serological data were normal, including liver and renal functions. 
On non-contrast enhanced CT, the tumor $(7.5 \times 6.5 \times$ $11.5 \mathrm{~cm}$ ) was found at the right abdominal wall, destroying the patient's right 11 th rib and extending outward from the body. The tumor presented slightly lower and heterogeneous attenuation, and it consisted of roughly two components: the lateral component, presenting nearly equal density to the surrounding muscles, and the medial component, presenting relatively low attenuation. Several broken bone fragments were seen inside the tumor, and invasion into the intercostal and latissimus dorsi muscles was suggested. Multiple metastases were also found in the bilateral lungs (Figure 1).

The FDG uptake in the lateral component of the tumor was much higher than that of the medial part, and the maximum standardized uptake value (SUVmax) of the lateral and medial components of the tumor were 7.1 and 2.0 , respectively. The SUVmax of the multiple lung nodules was less than 1.8 (Figure 2).

The MRI examination of the tumor showed heterogeneous higher signal intensity on T2-weighted images and lower signal intensity on T1-weighted images compared to the adjacent muscles. A gadolinium-enhanced fatsaturated T1-weighted image showed a heterogeneous marginal enhancement of the tumor. The diffusionweighted image appeared to be higher intensity in the lateral component compared to the medial component, and the lateral part of the tumor showed a lower apparent

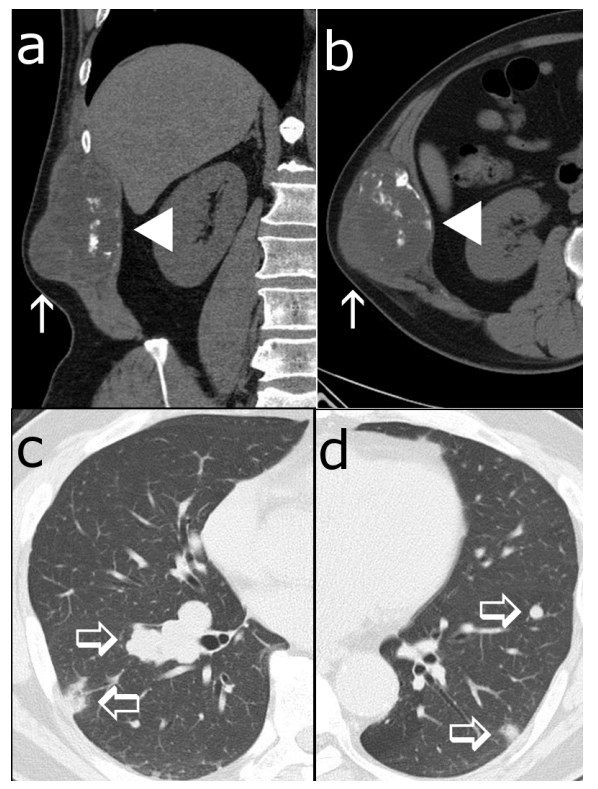

Figure 1. Non-contrast enhanced CT scans show a $7.5 \times 6.5$ $\times 11.5 \mathrm{~cm}$ tumor at the right abdominal wall of the patient, a 54-year-old male ((a), (b)). The lateral component of the tumor presented nearly equally but heterogeneous density to surrounding muscles (arrow), and the medial component presented slightly low attenuation with broken bone fragments (arrow head). Multiple metastases were found in the bilateral lungs ((c), (d); open arrows).
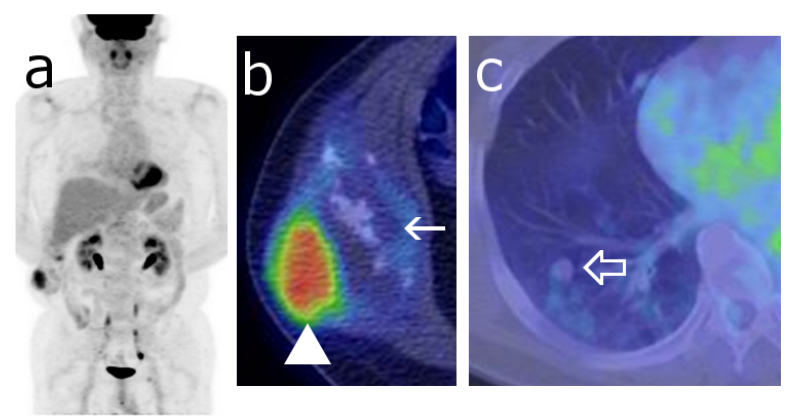

Figure 2. Maximum intensity projection (MIP) image (a) and fusion images ((b), (c)) of FDG-PET/CT showing the right abdominal wall tumor. The SUVmax of the tumor was measured as 7.1 at the lateral part of the tumor ((b) arrow head) and 2.0 at the medial component ((b) arrow). The FDG accumulation of the multiple lung metastases was weak (SUVmax < 1.8) ((c) open arrow).

diffusion coefficient compared to the medial component (Figure 3).

A biopsy was performed after the imaging examinations. The specimen of the tumor's lateral component showed cellular proliferation of spindle-shaped cells and less cellular myxoid stroma, accompanied by small slitlike vessels and chronic inflammatory infiltration. Scattered oval to short spindle-shaped cells with granular eosinophilic cytoplasm were also observed. With an immunostaining procedure, the spindle-shaped cells with eosinophilic cytoplasm were positive for desmin and myogenin and very focally positive for S-100 protein, but negative for alpha-SMA. The MIB-1 labeling index was $60 \%$ (Figure 4). These histological findings suggested MPNST with rhabdomyomatous differentiation, which is known as MTT.

Because the tumor invaded adjacent muscles and multiple lung metastases were found, chemotherapy rather than surgical tumor resection was selected for treatment. Although $100 \mathrm{mg} /$ body of adriamycin was administered, the tumor grew to $97 \times 71 \times 180 \mathrm{~mm}$ and the multiple lung metastases were much deteriorated. Three months after his admission, the patient died from respiratory failure caused by pneumonia.

\section{Discussion}

MTT is a very rare and highly progressive variant of MPNST with rhabdomyomatous differentiation. The first reported MTT case was described by Masson in 1932 [11]. Malignant triton tumor is named after the Triton salamander, which is able to regenerate limbs consisting of muscle, bone and nerve tissue if the cut end of the sciatic nerve is implanted into the soft tissue of its back. This regeneration was thought to be similar to the differentiation of muscular cell lines by malignant neural elements, as originally thought to occur in MTT $[1,12]$. 


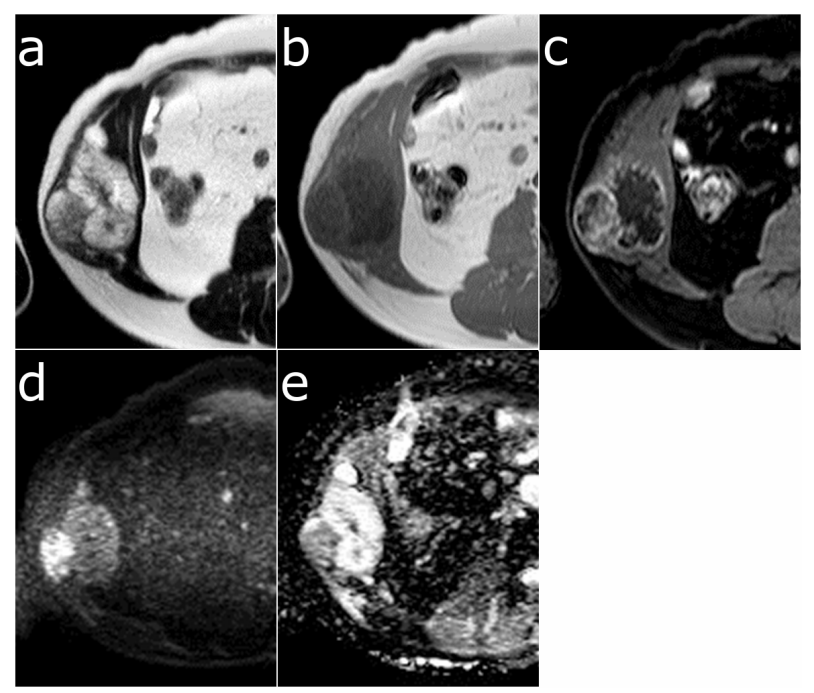

Figure 3. Axial MRI of the tumor showing heterogeneous high signal intensity on T2-weighted images (a) and low signal intensity on T1-weighted images (b). Gadoliniumenhanced fat-saturated T1-weighted image showed heterogeneous marginal enhancement of the tumor (c). The lateral component of the tumor, corresponding to high FDG accumulation, showed higher intensity on the diffusionweighted image (d) and a lower apparent diffusion coefficient (e) compared to the medial component.

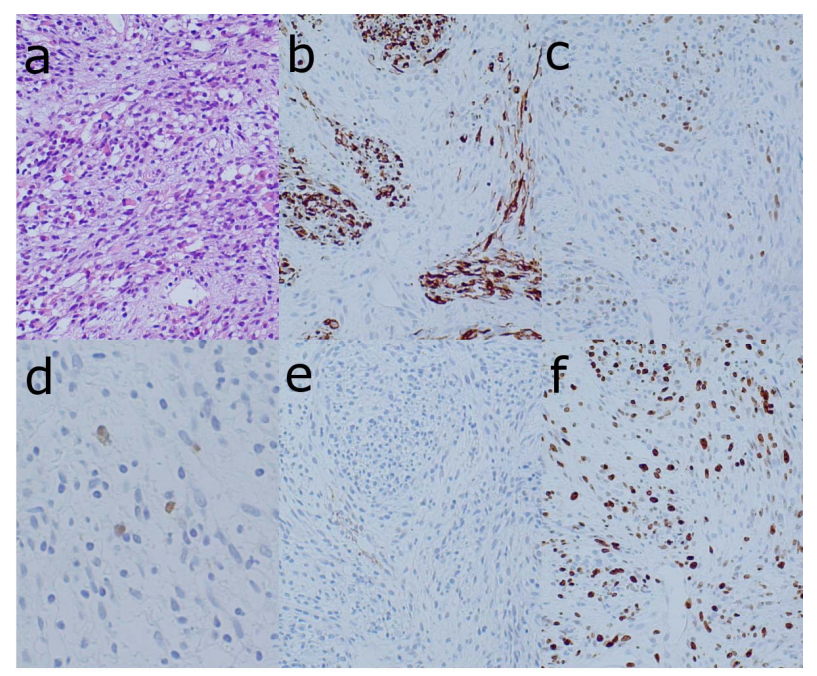

Figure 4. Photomicrographs of the specimen biopsied from the lateral component of the tumor showing the cellular proliferation of spindle-shaped cells with small slit-like vessels and chronic inflammatory infiltration and less cellular myxoid stroma ((a) HE $\times 100)$. Immunohistochemical stain of desmin $((b) \times 100)$ and myogenin $((c) \times 100)$ indicated the rhabdomyosarcomatous component of the tumor. Immunohistochemical stain of S-100 protein $((d) \times 200)$ showed that the spindle-shaped cells with eosinophilic cytoplasm were very focally positive, which indicates nerve sheath differentiation. No staining of alpha-SMA $((\mathrm{e}) \times \mathbf{1 0 0})$ indicated that there was no smooth muscle component in the tumor. The MIB-1 index was calculated as $\mathbf{6 0} \%$ from the staining with Ki-67 ((f) $\times 100)$.
Woodruff et al. reported that $57 \%$ of MTT patient had NF1 [2]. MTTs in patients with NF1, have shown a male predominance, young age (20 to 30 years old), and common presentation in the head and neck region. In contrast, MTT patients without NF1 show a female predominance, older age (30 to 50 years old), and tumors frequently located on the trunk, which agree with our case except for the gender $[13,14]$.

Woodruff et al. defined MTT with the following histopathological criteria: 1) the neoplasm is connected to peripheral nerves or occurs in patients with NF1;2) most of the neoplasm consists of Schwann cells; and 3) the neoplasm contains rhabdomyoblasts [15]. The biopsy specimen from our patient meets these criteria and was diagnosed as MTT.

MTT commonly presents heterogeneous CT attenuation and MR signal intensity because this tumor often contains necrosis, hemorrhage, degeneration, or cystic change. The contrast enhancement pattern of MTTs is also frequently heterogeneous [15]. These radiologic features are surely helpful for the diagnosis of MTT, but CT and MRI cannot reliably differentiate MTT from benign tumors, because the same features may also be seen in benign nerve sheath tumors $[6,16,17]$. Several studies demonstrated that FDG-PET was a sensitive and specific tool for distinguishing MPNST including MTT from benign peripheral nerve sheath tumors. The suggested cutoff values of SUVmax for the differentiation between malignant and benign peripheral nerve sheath tumor are $2.5-6.1$, which is a relatively wide-range with some degree of overlap [6,8-10]. To the best of our knowledge, three reports that include FDG-PET findings of MTT have been published [3-5].

Table 1 summarizes the characteristics of the seven MTT patients in the previous three reports and those of our patient, all of whom underwent FDG-PET or FDGPET/CT (males 3, females 5, age: 19 - 62, median 26.5). There were total of 11 lesions in these eight patients, and the SUVmax of the lesions ranged from 2.7 to 11.9 . Two patients did not have a history of NF1. Although SUVmax is affected by many factors including lesion size, applied FDG dose, and the time between the administration of FDG and the data acquisition, the intensity of FDG accumulation in these MTTs seems to be high, as is the case for MPNST [5]. It may be difficult to distinguish MTT from MPNST, even though FDG accumulation would be an indicative factor for the differentiation of MTT and benign peripheral nerve sheath tumor.

In our patient, we concluded that the lateral component of the tumor-which showed high FDG uptake-reflected the histopathological findings of cellular proliferation of spindle-shaped cells and less cellular myxoid stroma. The medial component of the tumor-which showed low FDG uptake - seemed to correspond to the myxoid tissue considering the MRI findings, but a histological verifica- 
Table 1. Reported MTT patients who underwent FDG-PET or FDG-PET/CT.

\begin{tabular}{ccccccc}
\hline & Age & Sex & $\begin{array}{c}\text { Max. dia. } \\
(\mathbf{c m})\end{array}$ & SUVmax & NF1 & Reference \\
\hline \multirow{2}{*}{25} & 25 & F & 26 & 10.8 & & \\
& & & 2.5 & 8.7 & $(-)$ & {$[3]$} \\
2 & 24 & M & 5.8 & 11.9 & $(+)$ & {$[3]$} \\
3 & 28 & F & 9 & 21 & $(+)$ & {$[4]$} \\
4 & 19 & F & Not shown & 2.7 & $(+)$ & {$[5]$} \\
5 & 23 & F & Not shown & 6.8 & $(+)$ & {$[5]$} \\
6 & 41 & F & Not shown & 8.4 & $(+)$ & {$[5]$} \\
7 & 62 & M & Not shown & 5.7 & $(+)$ & {$[5]$} \\
8 & 54 & M & 11.5 & 7.1 & $(-)$ & Our patient \\
\hline
\end{tabular}

tion of this was not obtained. Benz et al. suggested that the expression level of hypoxia-inducible factor- $1 \alpha$ (HIF$1 \alpha$ ) might also contribute to different glucose metabolic phenotypes in FDG-PET imaging [10]. However, the detailed mechanism underlying the FDG accumulation in this type of tumor has not been established. HIF-1 $\alpha$ was not measured in our patient.

Complete surgical resection with a wide margin and adjuvant radiotherapy are generally accepted in order to obtain the best outcome for an MTT. Systemic chemotherapy is selected if there is evidence of metastatic disease. The survival rates of patients with MTT are much lower than those of patients with MPNST. The reported crude 2- and 5-year survival rates of patients with MTT and MPNST are $15 \%$ and $11 \%$, versus $57 \%$ and $39 \%$, respectively $[18,19]$.

\section{Conclusions}

Our patient's MTT showed high FDG accumulation. In the light of the histopathological findings, the high FDG uptake seemed to correlate with the proliferation of spindle-shaped cells and less cellular myxoid stroma.

Although it is still difficult to distinguish MTT from MPNST, FDG-PET/CT can be helpful tool for differentiation between MTT from benign peripheral nerve sheath tumor.

\section{REFERENCES}

[1] J. S. J. Brooks, "Disorders of Soft Tissue," In: S. S. Sternberg, Ed., Diagnostic Surgical Pathology, 3rd Edition, Lippincott Williams and Wilkins, Philadelphia, 1999, pp. 131-221.

[2] J. M. Woodruff, H. P. Kourea, D. N. Louis and B. W. Scheithauer, "Malignant Peripheral Nerve Sheath Tumor (MPNST)," In: P. Kleihues and W. K. Cavenee, Eds., Pathology and Genetics of Tumours of the Nervous Sys- tem, IARC Press, Lyon, 2000, pp. 172-174.

[3] N. H. Thoennissen, C. Schliemann, U. Brunnberg, E. Schmidt, A. Staebler, L. Stegger, C. Bremer, C. Schleicher, R. M. Mesters, C. Müller-Tidow and W. E. Berdel, "Chemotherapy in Metastatic Malignant Triton Tumor: Report on Two Cases," Oncology Reports, Vol. 18, No. 4, 2007, pp. 763-767.

[4] J. Dartnell, J. Pilling, R. Ferner, P. Cane and L. LangLazdunski, "Malignant Triton Tumor of the Brachial Plexus Invading the Left Thoracic Inlet: A Rare Differntial Diagnosis of Pancoast Tumor," Journal of Thoracic Oncology, Vol. 4, No. 1, 2009, pp. 135-137. doi:10.1097/JTO.0b013e31819151ab

[5] R. E. Ferner, J. D. Lucas, M. J. O’Doherty, R. A. Hughes, M. A. Smith, B. F. Cronin and J. Bingham, "Evaluation of (18)fluorodeoxyglucose Positron Emission Tomography ((18)FDG PET) in the Detection of Malignant Peripheral Nerve Sheath Tumours Arising from within Plexiform Neurofibromas in Neurofibromatosis 1," Journal of Neurology, Neurosurgery and Psychiatry, Vol. 68, No. 3, 2000, pp. 353-357. doi:10.1136/jnnp.68.3.353

[6] R. E. Ferner, J. F. Golding, M. Smith, E. Calonje, W. Jan, V. Sanjayanathan and M. O'Doherty, "[18F]2-Fluoro-2Deoxy-D-Glucose Positron Emission Tomography (FDG PET) as a Diagnostic Tool for Neurofibromatosis 1 (NF1) Associated Malignant Peripheral Nerve Sheath Tumours (MPNSTs): A Long-Term Clinical Study," Annals of Oncology, Vol. 19, No. 2, 2008, pp. 390-394. doi:10.1093/annonc/mdm450

[7] W. Brenner, R. E. Friedrich, K. A. Gawad, C. Hagel, A. von Deimling, M. de Wit, R. Buchert, M. Clausen and V. F. Mautner, "Prognostic Relevance of FDG PET in Patients with Neurofibromatosis Type-1 and Malignant Peripheral Nerve Sheath Tumors," European Journal of Nuclear Medicine and Molecular Imaging, Vol. 33, No. 4, 2006, pp. 428-432. doi:10.1007/s00259-005-0030-1

[8] M. Charest, M. Hickeson, R. Lisbona, J. A. Novales-Diaz, V. Derbekyan and R. E. Turcotte, "FDG-PET/CT Imaging in Primary Osseous and Soft Tissue Sarcomas: A Retrospective Review of 212 Cases," European Journal of Nuclear Medicine and Molecular Imaging, Vol. 36, No. 12, 2009, pp. 1944-1951. doi:10.1007/s00259-009-1203-0

[9] V. S. Warbey, R. E. Ferner, J. T. Dunn, E. Calonje and M. J. O'Doherty, "[18F]FDG-PET/CT in the Diagnosis of Malignant Peripheral Nerve Sheath Tumours in Neurofibromatosis Type-1," European Journal of Nuclear Medicine and Molecular Imaging, Vol. 36, No. 5, 2009, pp. 751-757. doi:10.1007/s00259-008-1038-0

[10] M. R. Benz, J. Czernin, S. M. Dry, W. D. Tap, M. S. Allen-Auerbach, D. Elashoff, M. E. Phelps, W. A. Weber and F. C. Eilber, "Quantitative F18-Fluorodeoxyglucose Positron Emission Tomography Accurately Characterizes Peripheral Nerve Sheath Tumors as Malignant or Benign," Cancer, Vol. 116, No. 2, 2010, pp. 451-458. doi:10.1002/cncr.24755

[11] P. Masson, "Recklinghausen's Neurofibromatosis, Sensory Neuromas and Motor Neuromas," The International Press, New York, 1932.

[12] A. K. Bose, A. P. Deodhar and A. J. Duncan, "Malignant 
Triton Tumor of the Right Vagus," The Annals of Thoracic Surgery, Vol. 74, No. 4, 2002, pp. 1227-1228. doi:10.1016/S0003-4975(02)03789-X

[13] E. Aldlyami, A. Dramis, R. J. Grimer, A. Abudu, S. R. Carter and R. M. Tillman, "Malignant Triton Tumor of the Thigh-A Retrospective Analysis of Nine Cases," Europen Journal of Surgical Oncology, Vol. 32, No. 7, 2006, pp. 808-810. doi:10.1016/j.ejso.2006.04.008

[14] J. S. Brooks, M. Freeman and H. T. Enterline, "Malignant 'Triton' Tumors. Natural History and Immunohistochemistry of Nine New Cases with Literature Review," Cancer, Vol. 55, No. 11, 1985, pp. 2543-2549. doi:10.1002/1097-0142(19850601)55:11<2543::AID-CN CR2820551105>3.0.CO;2-4

[15] J. M. Woodruff, N. L. Chernik, M. C. Smith, W. B. Millett and F. W. Jr. Foote, "Peripheral Nerve Tumors with Rhabdomyosarcomatous Differentiation (Malignant 'Triton' Tumors)," Cancer, Vol. 32, No. 2, 1973, pp. 426-439. doi:10.1002/1097-0142(197308)32:2<426::AID-CNCR28 20320221>3.0.CO;2-W

[16] A. D. Levy, N. Patel, N. Dow, R. M. Abbott, M. Miettinen and L. H. Sobin, "From the Archives of the AFIP:
Abdominal Neoplasms in Patients with Neurofibromatosis Type 1: Radiologic-Pathologic Correlation," RadioGraphics, Vol. 25, No. 2, 2005, pp. 455-480. doi:10.1148/rg.252045176

[17] G. Hermann, I. F. Abdelwahab, T. T. Miller, M. J. Klein and M. M. Lewis, "Tumour and Tumour-Like Conditions of the Soft Tissue: Magnetic Resonance Imaging Features Differentiating Benign from Malignant Masses," British Journal of Radiology, Vol. 65, No. 769, 1992, pp. 14-20. doi:10.1259/0007-1285-65-769-14

[18] J. M. Woodruff and G. Perino, "Non-Germ-Cell of Teratomatous Malignant Tumors Showing Additional Rhabdomyoblastic Differentiation, with Emphasis on the Malignant Triton Tumor," Seminars in Diagnostic Pathology, Vol. 11, No. 1, 1994, pp. 69-81.

[19] R. H. Hruban, M. H. Shiu, R. T. Senie and J. M. Woodruff, "Malignant Peripheral Nerve Sheath Tumors of the Buttock and Lower Extremity: A Study of 43 Cases," Cancer, Vol. 66, No. 6, 1990, pp. 1253-1265. doi:10.1002/1097-0142(19900915)66:6<1253::AID-CNC $\underline{\text { R2820660627>3.0.CO;2-R }}$ 